\title{
ROBUST AND ADAPTIVE TESTS FOR THE TWO-SAMPLE LOCATION PROBLEM
}

\author{
Herbert Büning, FU Berlin
}

In the important two-sample location problem the application of the $t-$ test depends on very restrictive assumptions such as normality and equal variances of the two random variables $X$ and $Y$ and it is wellknown that the $t$-test is not robust with respect to level $\alpha$ or power $\beta$ in the case of departures from normality and for unequal variances. Thus if the assumptions of the t-test are not satisfied it is more appropriate to apply a robust version of the $t$-test, like the trimmed $t-$ test, or a nonparametric test, like the wilcoxon. But the choice of a suitable robust or nonparametric test also depends on the underlying distribution, which may be symmetric or asymmetric and may have light, middle or heavy tails. Since in most cases the practising statistician has no clear idea about the underlying distribution an adaptive test should be applied which takes the given data set into account. Such real data sets are presented in order to demonstrate departures from tile model of the t-test and to suggest the application of a roibust or ariaptive test. Some of these tests are discussed and comparid with each other and with the classical t-test under different models of nonnormality, like heavy-tailed or asymmetric distributions. It is shown that an adaptive test behaves well over a broad class of distribution functions. Although it is not always the best one, in most cases it is second best for each of the distributions considered. But this is just the philosophy of an adaptive test. It works in the sense of the "safety first" principle.

Hogg, R. V. (1976). A new dimension to nonparametric tests. Commun. Stat., Ser. A, Vol.5, $1313-1325$

Büning, H, (1991). Robuste und adaptive Tests. De Gruyter, Berlin. 